\title{
Alcohol, cirrosis y predisposición genética
}

\author{
Alcohol, Cirrhosis, and Genetic Predisposition
}

Mónica Marcela Gaviria C., Biol., MSc (c), ${ }^{1}$ Gonzalo Correa Arango, MD, ${ }^{1}$ María Cristina Navas N., MSc, PhD. ${ }^{1}$

1 Grupo de Gastrohepatología, Facultad de Medicina, Universidad de Antioquia, UdeA. Medellín, Colombia.

Fecha recibido: $15-12-14$

Fecha aceptado: 26-01-16

\begin{abstract}
Resumen
La cirrosis hepática es la tercera causa de muerte alrededor del mundo que es atribuible al consumo de alcohol. Más del $80 \%$ de los consumidores crónicos de alcohol desarrollan esteatosis y entre el $20 \%$ al $40 \%$ presentan otras complicaciones como fibrosis, hepatitis alcohólica y cirrosis; sin embargo, no todos los individuos con consumo crónico de alcohol desarrollan cirrosis, en parte debido al componente genético de cada individuo. El grado de actividad de las enzimas que metabolizan el alcohol está influenciado por polimorfismos presentes en los genes que codifican para estas enzimas, y corresponde a uno de los factores determinantes para el desarrollo de una hepatopatía terminal en respuesta al consumo de alcohol. Entre las enzimas implicadas en el metabolismo del alcohol están la alcohol deshidrogenasa (ADH), el citocromo P450 2E1 (CYP2E1) y la acetaldehído deshidrogenasa (ALDH), de las cuales se ha reportado que la mayor actividad de ADH y CYP2E1 y la menor actividad de ALDH pueden conferir riesgo en algunas poblaciones por la acumulación de acetaldehído, el cual es tóxico para el organismo.

Se realizó una revisión en la literatura de los principales aspectos del metabolismo del alcohol y polimorfismos (genotipos) de enzimas que intervienen en el metabolismo del alcohol como factor de riesgo. Esto se hizo mediante la búsqueda de material bibliográfico a través de la base de datos PubMed desde 1990 hasta el 2013 utilizando las palabras claves alcohol liver disease, ADH, ALDH, CYP2E1 y polymorphism.
\end{abstract}

\section{Palabras clave}

Hepatopatía alcohólica, ADH, ALDH, CYP2E1, polimorfismo.

\begin{abstract}
Liver cirrhosis is the third most common cause of death attributable to alcohol consumption throughout the world. More than $80 \%$ of chronic drinkers develop steatosis, and $20 \%$ to $40 \%$ develop other complications such as fibrosis, alcoholic hepatitis and cirrhosis. However, not everyone who chronically consumes alcohol develops cirrhosis. This is partly because of the genetic component of each individual. The level of activity of the enzymes that metabolize alcohol is influenced by polymorphisms of the genes that coding for these enzymes. This is one of the determining factors in the development of terminal liver disease in response to alcohol consumption. Among the enzymes involved in alcohol metabolism are alcohol dehydrogenase (ADH), cytochrome P450 2E1 (CYP2E1) and acetaldehyde dehydrogenase (ALDH). It has been reported that higher levels of activity of ADH and CYP2E1 and lower levels of activity of ALDH may be risk factors in some populations for accumulation of acetaldehyde which is toxic for the organism.

This literature review covers the most important aspects of alcohol metabolism including polymorphisms (genotypes) of enzymes involved in the metabolism of alcohol as a risk factor. A search through the PubMed database from 1990 to be held 2013 was conducted using the keywords alcoholic liver disease, ADH, ALDH, CYP2E1, and polymorphism.
\end{abstract}

\section{Keywords}

Alcoholic liver disease, ADH, ALDH, CYP2E1, polymorphism. 


\section{INTRODUCCIÓN}

El consumo crónico de alcohol es la causa de 3,3 millones de muertes alrededor del mundo, que corresponde al 5,9\% del total anual, siendo 7,6\% en varones y $4,0 \%$ en mujeres; asimismo, es uno de los factores de riesgo con mayor morbilidad y mortalidad en enfermedad general. Adicionalmente, se le atribuye el 5,1\% de la carga global e invalidez (1).

Existe evidencia de la relación causal entre el consumo de alcohol y por lo menos 200 enfermedades como gastritis, pancreatitis, enfermedad cardiovascular, cirrosis hepática, carcinoma hepatocelular, cáncer gástrico, entre otros; las patologías asociadas con el consumo crónico de alcohol son determinadas por el volumen consumido, el patrón de bebida y la calidad del alcohol ingerido $(1,2)$. El metabolismo del alcohol es un proceso complejo que implica absorción, distribución y eliminación; en el hígado se metaboliza más del 90\% del alcohol en el organismo, se convierte en acetaldehído mediante la acción de las enzimas alcohol deshidrogenasa (ADH), citocromo P540-2E1 (CYP2E1) o catalasa; luego el acetaldehído se convierte en acetato y agua por la enzima aldehído deshidrogenasa (ALDH) (3).
Se ha demostrado in vitro que algunos polimorfismos en los genes que codifican las enzimas $\mathrm{ADH}, \mathrm{CYP} 2 \mathrm{E} 1 \mathrm{y}$ ALDH estarían asociados con mayor actividad enzimática y con la acumulación de metabolitos, como el acetaldehído, que presenta un efecto tóxico en el organismo y produce daño en el tejido hepático (4-6).

\section{METABOLISMO DEL ALCOHOL}

El etanol se absorbe por el tracto intestinal para ser transportado al hígado, donde se metaboliza el 90\% del alcohol; el 2\% al $10 \%$ restante se metaboliza en los pulmones y riñones $(3,7)$.

En el metabolismo del alcohol en el hígado intervienen 3 sistemas (figura 1). El más importante es la $\mathrm{ADH}$; esta enzima está en el citosol de los hepatocitos y cataliza la formación de acetaldehído por transferencia del hidrógeno del grupo $\mathrm{OH}$ al cofactor nicotinamida adenina dinucleótido (NAD) para convertirlo en NADH y luego, por transhidrogenación, en $\operatorname{NADPH}(7,8)$. Durante la oxidación del acetaldehído a acetato por la enzima aldehído deshidrogenasa $(\mathrm{ALDH})$ se produce un exceso de $\mathrm{NADH}$ que incrementa la relación $\mathrm{NADH} / \mathrm{NAD}$ y tiene efectos en el metabolismo de los carbohidratos y lípidos; el $\mathrm{NADH}$ interfiere con el

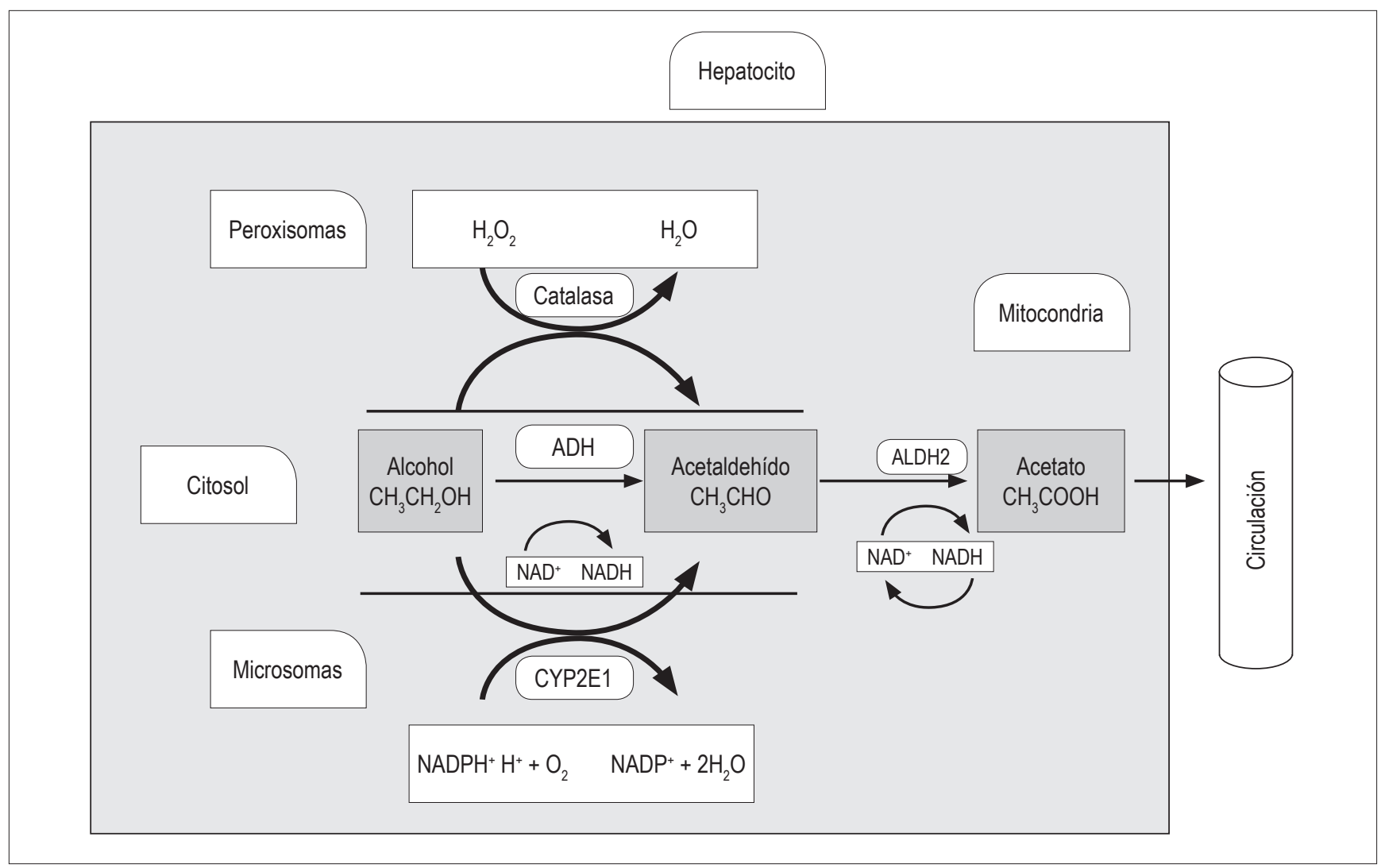

Figura 1. Metabolismo oxidativo del alcohol. ADH: alcohol deshidrogenasa; CYP2E1: citocromo P450 subfamilia 2E1; ALDH: aldehído deshidrogenasa. 
transporte de ácidos grasos libres (AGL) y facilita la formación de ácidos grasos esterificados, ya que los ácidos grasos estarían reaccionando con el alcohol, el cual extrae 1 hidrógeno de 1 ácido graso poliinsaturado, lo que lleva a la degradación. El exceso de NADH limitaría la disponibilidad del NAD necesario para el transporte de los AGL (9). El acetato se incorpora en el ciclo de Krebs como acetil coenzima A (acetil CoA) y en caso de no transferirse al ciclo, su acumulación puede resultar en la producción de cuerpos cetónicos, ocasionando cetonemia y cetonuria $(7,9-13)$.

El segundo sistema que interviene es el microsomal de oxidación del etanol (MEOS), un sistema inducible en el que participa el citocromo P450 (CYP450). Específicamente, el CYP2E1 cumple una función principal metabólica en los microsomas del hígado; la transcripción de este gen se activa en condiciones de alto consumo de alcohol, se metaboliza a acetaldehído utilizando el NAD fosforilado o el NAD reducido $(\mathrm{NADPH})$ y oxígeno $\left(\mathrm{O}_{2}\right)$. Este sistema contribuye con el 3\% al 8\% del metabolismo del alcohol (14).

El tercer sistema funciona en los peroxisomas de la célula hepática mediante la actividad de la catalasa, que metaboliza el alcohol a acetaldehído a través de la peroxidación, en presencia de peróxido de hidrogeno $\left(\mathrm{H}_{2} \mathrm{O}_{2}\right)$, que luego se transforma en agua. Este sistema metaboliza menos del $2 \%$ del alcohol ingerido (3).

\section{ALCOHOL COMO FACTOR DE RIESGO}

El consumo crónico de alcohol es el factor de riesgo del $20 \%$ al $50 \%$ de los casos de cirrosis hepática a nivel mundial (1); en el año 2010, la cirrosis hepática atribuible al alcohol fue responsable del $47,9 \%$ de las muertes por esta hepatopatía (15). El 80\% al $90 \%$ de los consumidores crónicos de alcohol desarrollan hígado graso y están en riesgo de presentar complicaciones como esteatohepatitis, fibrosis, hepatitis alcohólica, cirrosis alcohólica, cirrosis caracterizada por fibrosis y distorsión de la arquitectura normal del hígado, y hepatocarcinoma (figura 2) $(7,16)$.

Se ha establecido, en estudios de cohorte y estudios de casos y controles, que el consumo de $>30$ gramos/ día (g/d) (teniendo en cuenta que la OMS señala un valor aproximado de 10 gramos de etanol por trago de bebida destilada, vino o cerveza) de etanol incrementa 11 veces el riesgo de cirrosis (OR: 10,9, IC 95\%: 3,6-33,5) y el consumo de 60 $\mathrm{g} / \mathrm{d}$ de etanol por un periodo de 5 años incrementa 7 veces el riesgo de carcinoma hepatocelular (CHC) (OR: 7,0, IC 95\%: 4,5-11,1)(17-19).

En modelos animales se ha demostrado que el consumo crónico de alcohol afecta el transporte de vitaminas en el intestino delgado; esto se comprobó en ratas Sprague Dawley, que se alimentaron por 6 a 8 semanas con caseína, vitaminas, minerales, maltosa y altas concentraciones de tiamina y alcohol. El alcohol disminuyó el transporte de tiamina y la actividad de la bomba Na-K (20). Asimismo, tras un seguimiento de 6 años se observó a primates babuinos (Papio hamadryas) que ingirieron alcohol y desarrollaron el espectro de enfermedades hepáticas como esteatosis, fibrosis hepática y cirrosis; mientras que los que no consumieron alcohol no desarrollaron ninguna hepatopatía, lo cual demostró que el alcohol aceleraba el proceso de fibrosis, llevando al desarrollo de cirrosis (21-23).

Igualmente, en humanos se han realizado estudios para determinar el riesgo de desarrollar cirrosis por el consumo de alcohol y se ha concluido que el 50\% de la variación fenotípica, es decir el desarrollo de enfermedad hepática, puede ser atribuida al factor genético; se ha observado

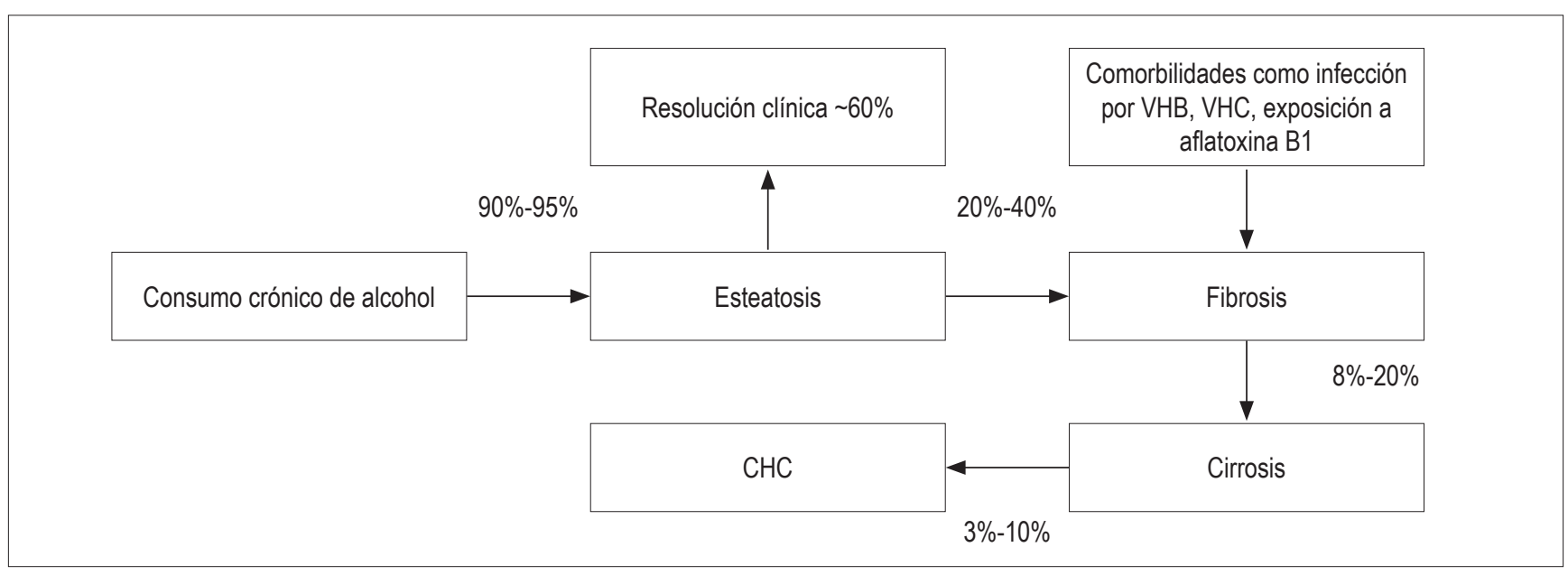

Figura 2. Hepatopatías asociadas con el abuso crónico de alcohol. VHB: virus de la hepatitis B; VHC: virus de la hepatitis C; CHC: carcinoma hepatocelular. 
que gemelos monocigóticos progresan a cirrosis más fácilmente por el consumo de alcohol, comparado con gemelos dicigóticos $(24,25)$.

Adicionalmente, se ha demostrado interacción entre el consumo de alcohol y la infección por virus de hepatitis B (VHB) y C (VHC). Durante un seguimiento de 5 años, el riesgo de desarrollar $\mathrm{CHC}$ en consumidores de alcohol y con infección viral se duplicó respecto de consumidores de alcohol $(60 \mathrm{~g} / \mathrm{d})$ sin infección por VHB o VHC (17). También se ha demostrado que pacientes con cirrosis (diagnóstico realizado por histología, laboratorio e imagen) y que consumen alcohol presentan un riesgo 24 veces mayor (OR: 23,8 , IC 95\%: 7,3-7,9) de desarrollar CHC comparado con pacientes sin enfermedad hepática. Adicionalmente, se analizó el efecto sinérgico del alcohol, la obesidad y el tabaquismo y se observó un riesgo de 7,4 veces (OR: 7,4, IC 95\%: 2,1-14,6) de desarrollar CHC comparado con los casos que solo presentaban un factor de riesgo (26).

\section{ENZIMAS IMPLICADAS EN EL METABOLISMO DEL ALCOHOL}

\section{Alcohol deshidrogenasa (ADH)}

Esta enzima está conformada por 2 subunidades codificadas por los genes $\mathrm{ADH} 1, \mathrm{ADH} 4, \mathrm{ADH} 5, \mathrm{ADH} 6$ y $\mathrm{ADH} 7$, ubicados en el brazo largo del cromosoma 4 (4q21-24)(27, 28). Estos genes codifican diferentes subunidades de la $\mathrm{ADH}$ hepática $(\alpha, \beta, \gamma, \pi, \chi)$; las subunidades determinan las 12 isoenzimas (14). Las isoenzimas se han agrupado en 5 clases (I-V) (tabla 1); en el hígado están presentes las clases I a III (29), siendo la clase I la predominante con 3 isoenzimas $(\mathrm{ADH} 1 \mathrm{~A}, \mathrm{ADH} 1 \mathrm{~B}, \mathrm{ADH} 1 \mathrm{C})$ que presentan homología mayor al $80 \%$ en las secuencias $(30,31)$.

Tabla 1. Isoenzimas de la alcohol deshidrogenasa (ADH).

\begin{tabular}{|c|c|c|c|c|}
\hline Clase & Gen & Alelo & Subunidad & Tejido \\
\hline \multirow[t]{6}{*}{1} & ADH1A & ADH1A & $\mathrm{aa}$ & Hígado \\
\hline & ADH1B & ADH1B*1 & $\beta 1 \beta 1$ & Hígado, pulmón \\
\hline & & $\mathrm{ADH} 1 \mathrm{~B}^{*} 2$ & $\beta 2 \beta 2$ & \\
\hline & & $\mathrm{ADH} 1 \mathrm{~B}^{*} 3$ & $\beta 3 \beta 3$ & \\
\hline & ADH1C & ADH1C*1 & ү1ү1 & Hígado, estómago \\
\hline & & ADH $1 C^{*} 2$ & ү2ү2 & \\
\hline ॥ & $\mathrm{ADH} 4$ & $\mathrm{ADH} 4$ & $\Pi$ & Hígado, córnea \\
\hline III & ADH5 & ADH5 & $x$ & Varios tejidos \\
\hline IV & ADH7 & ADH7 & $\mu$ & Estómago \\
\hline V & ADH6 & ADH6 & $-^{*}$ & Estómago \\
\hline
\end{tabular}

*Para ADH6 no se reporta subunidad.
La $\mathrm{ADH}$ es una enzima de $40 \mathrm{kDa}$ (kilo Dalton), dimérica, que contiene zinc y es dependiente de NAD (32); pertenece a la familia de enzimas deshidrogenasas-reductasas que catalizan la oxidación de alcohol para producir aldehído o cetona (33). Las más representativas en el hígado son la clase tipo I (ADH1A, $\mathrm{ADH} 1 \mathrm{~B}, \mathrm{ADH} 1 \mathrm{C})$, la clase II está presente en bajo nivel en el hígado y la clase III se encuentra en todos los tejidos pero está poco asociada con el metabolismo del alcohol $(33,34)$. La ADH1A predomina en el primer trimestre del desarrollo fetal, luego del nacimiento se presenta mayor expresión de $\mathrm{ADH} 1 \mathrm{~B}$ y $\mathrm{ADH} 1 \mathrm{C}(35,36)$.

La isoenzima $\mathrm{ADH} 1 \mathrm{~B}$ tiene el alelo $\mathrm{ADH}_{1} \mathrm{~B}^{*} 1(\beta 1 \beta 1)$ o tipo silvestre, presente en más del $90 \%$ de población caucásica; $\mathrm{ADH}_{1} \mathrm{~B}^{*} 2(\beta 2 \beta 2)$ se reporta con alta frecuencia en población asiática $(\sim 70 \%)$ y $\mathrm{ADH}_{1} * 3(\beta 3 \beta 3)$ es común en población africana $(\sim 16 \%)(37)$. Las isoenzimas $\mathrm{ADH} 1 \mathrm{~B}$ y $\mathrm{ADH} 1 \mathrm{C}$ presentan variaciones en su cadena de aminoácido generando enzimas con mayor actividad catalítica (tabla 2).

Tabla 2. Polimorfismos en el gen $\mathrm{ADH} 1$ y alteración de función relacionada.

\begin{tabular}{|c|c|c|c|}
\hline Alelo & $\begin{array}{l}\text { Polimorfismo } \\
\text { secuencia } \\
\text { nucleótido }\end{array}$ & $\begin{array}{l}\text { Polimorfismo } \\
\text { en secuencias } \\
\text { aminoácido }\end{array}$ & $\begin{array}{l}\text { Alteración de } \\
\text { función }\end{array}$ \\
\hline $\mathrm{ADH} 1 \mathrm{~B}^{\star 2} 2$ & $\begin{array}{l}\text { Transición } G \rightarrow A \\
\text { en la posición } \\
100458342\end{array}$ & $\begin{array}{l}\text { Arg } \rightarrow \text { His } \\
\text { posición } 47\end{array}$ & \multirow{2}{*}{$\begin{array}{l}\text { Ambas } \\
\text { presentan mayo } \\
\text { actividad (20 } \\
\text { veces) catalítica } \\
\text { respecto de } \\
\text { la silvestre } \\
(\text { ADH1B*1) }\end{array}$} \\
\hline $\mathrm{ADH} 1 \mathrm{~B}^{*} 3$ & $\begin{array}{l}\text { Transición de } \mathrm{C} \rightarrow \mathrm{T} \\
\text { en la posición } \\
100229017\end{array}$ & $\begin{array}{l}\text { Arg } \rightarrow \text { Cys } \\
\text { posición } 369\end{array}$ & \\
\hline $\mathrm{ADH} 1 \mathrm{C}^{\star} 2$ & $\begin{array}{l}\text { Sustitución } C \rightarrow T \\
\text { en la posición } \\
100482988 \\
\text { Sustitución de } \\
A \rightarrow G \text {, en posición } \\
100479812\end{array}$ & $\begin{array}{l}\mathrm{Arg} \rightarrow \mathrm{Gln} \\
\text { posición } 272 \\
\mathrm{lle} \rightarrow \text { Val } \\
\text { posición } 350\end{array}$ & $\begin{array}{l}\text { ADH1C*1 } \\
\text { (silvestre) } \\
\text { metaboliza } \\
2,5 \text { veces más } \\
\text { rápido que } \\
\text { ADH1C*2 }\end{array}$ \\
\hline
\end{tabular}

\section{Aldehído deshidrogenasa (ALDH)}

La aldehído deshidrogenasa (ALDH) es una súper familia de genes que codifican para proteínas que catalizan la conversión de sustratos de aldehído a carboxilatos vía NAD+ $(38,39)$. Su forma citosólica se codifica por el gen ALDH1 ubicado en el brazo largo del cromosoma 9 en la región 9q21.13 (40). La forma mitocondrial se codifica por el gen ALDH2 ubicado en el brazo largo del cromosoma 12 en la región 12q24.12; la ALDH2 presenta mayor importancia, ya que metaboliza mayor cantidad de acetaldehído $(14,40$, $41)$. 
$\mathrm{La}$ ALDH2 es una enzima mitocondrial de $54 \mathrm{kDa}$ con una estructura tetramérica que presenta actividad catalítica para la oxidación de acetaldehído; oxida más del $90 \%$ del acetaldehído producido por la oxidación del etanol en la desintoxicación del hígado $(14,42)$. En el gen de ALDH2 se han descrito 2 polimorfismos, ALDH2* 1 de alta actividad y ALDH2* 2 que presenta una mutación en el aminoácido 487 (sustitución Glu $\rightarrow$ Lys); esta mutación está asociada con baja actividad específica y por tanto, la oxidación del acetaldehído es menos eficiente, generando la acumulación de este metabolito $(41,43)$. Esto causa diferentes efectos tóxicos en el hígado y la alteración de proteínas en el hepatocito por unión del compuesto al grupo amino de las proteínas (figura 1) $(3,42)$.

La población caucásica presenta las 2 isoenzimas, ALDH1 y ALDH2. El alelo de ALDH2 descrito en esta población no presenta la mutación asociada con disminución de la capacidad de la enzima para metabolizar el acetaldehído. Aproximadamente el 50\% de la población japonesa pierde la actividad de la isoenzima ALDH2, esto debido a la transición $\mathrm{G} / \mathrm{C} \rightarrow \mathrm{A} / \mathrm{T}$ en el exón 12, con la sustitución del aminoácido Glu $\rightarrow$ Lys en la posición 14 del $\mathrm{COOH}$ terminal, la cual genera una proteína defectuosa en el sitio catalítico y por tanto, presenta actividad metabólica disminuida (38).

\section{Citocromo P450 (CYP2E1)}

La familia del CYP450 tiene como función principal el metabolismo de diferentes xenobióticos que ingresan al cuerpo, como medicamentos, drogas y alcohol; se divide en 44 subfamilias, de las cuales la IIE es la más importante en el metabolismo del alcohol. Esta subfamilia se codifica por el gen CYP2E1, localizado en el cromosoma 10 en la región 10q26.3 (44). El gen CYP2E1 codifica una enzima de $56,9 \mathrm{kDa}$ con capacidad de metabolizar fármacos, hormonas y toxinas xenobióticas como el etanol; la enzima está localizada en la membrana del retículo endoplasmático y presenta funciones de oxidorreducción $(6,44-46)$.

En situaciones de consumo crónico de alcohol, el gen presenta una alta tasa de transcripción puesto que es un sistema inducible por el etanol. El CYP2E1 participa en el sistema MEOS ubicado en los microsomas de la célula, como el retículo (figura 1), cuya función es metabolizar el etanol a acetaldehído; durante este proceso se producen especies reactivas de oxígeno (ROS) $(47,48)$.

Se ha reportado relación entre el nivel de expresión de CYP450 y la cantidad de aductos en el ADN por la unión de compuestos como acetaldehído y bases modificadas, los cuales son conocidos por su capacidad de inducir una mutación en el $\mathrm{ADN}$ que facilite la activación de oncogenes e inactive genes supresores de tumores, o induzca respuesta inmune por el reconocimiento de proteínas con uniones de estructuras foráneas que sean reconocidas por el sistema inmune $(6,49)$.

Entre los polimorfismos estudiados de CYP2E1, el CYP2E1*5B corresponde a un cambio de secuencia de la región reguladora 5 del gen (C-1053T); este cambio se encuentra asociado con una mayor tasa de transcripción y de actividad enzimática, y se ha reportado con el aumento del riesgo de hepatopatías asociadas con el consumo de alcohol (44).

\section{Catalasa}

El gen que codifica para la catalasa está ubicado en el cromosoma 11, en la región 11 p13 (50). La catalasa es una proteína oligomérica con 4 subunidades de $60 \mathrm{kDa}$ (14). El sistema catalasa (figura 1) se localiza en los peroxisomas y su función principal es regular los niveles de peróxido de hidrogeno y la peroxidación del etanol a acetaldehído en presencia de $\mathrm{H}_{2} \mathrm{O}_{2}(51)$.

La catalasa presenta varias isoformas: la tipo I se caracteriza por un cambio de $\mathrm{G} \rightarrow \mathrm{A}$ en la posición 5 del intrón 4; la tipo II presenta una deleción en la posición 358 de $\mathrm{T}$ en el exón 4; y la tipo III presenta una inserción en la posición 138GA en el exón $2(52-, 54)$. Estas mutaciones están asociadas con la disminución de la actividad catalítica y por tanto, con el metabolismo disminuido $(<10 \%)$ de peróxido de hidrógeno; esto ocasiona acatalasemia, un síndrome recesivo autosómico en el cual está disminuida la actividad de la catalasa en eritrocitos (54).

\section{ALCOHOL Y CIRROSIS: FISIOPATOLOGÍA}

El acetaldehído, metabolito del alcohol, se acumula produciendo daño hepático por su capacidad para generar la formación de aductos en el ADN en sitios apúrinicos y apirimidínicos (figura 3). Igualmente, la generación de ROS durante el metabolismo del alcohol por la enzima CYP2E1, compuestos en donde se incluyen peróxido de hidrógeno $\left(\mathrm{H}_{2} \mathrm{O}_{2}\right)$, superóxidos y radical hidroxilo, tiene un papel importante por la capacidad de generar daño, tanto en el $\mathrm{ADN}$ como en la oxidación de ácidos grasos (6).

El acetaldehído presenta efectos tóxicos, como daño en la mitocondria por la alteración en la membrana celular; daño al $\mathrm{ADN}$, lo que reduce la utilización de oxígeno por las mismas; muerte celular por disminución de la actividad enzimática de proteínas capaces de degradar ROS, como la glutatión $s$ transferasa (GST) y la peroxidación de lípidos (7, 9-13).

Debido a la peroxidación de lípidos, en la generación se da la formación de la esteatosis hepática como consecuencia del abuso de alcohol, caracterizada por la acumulación de grasa en vacuolas de distinto tamaño en el citoplasma de 


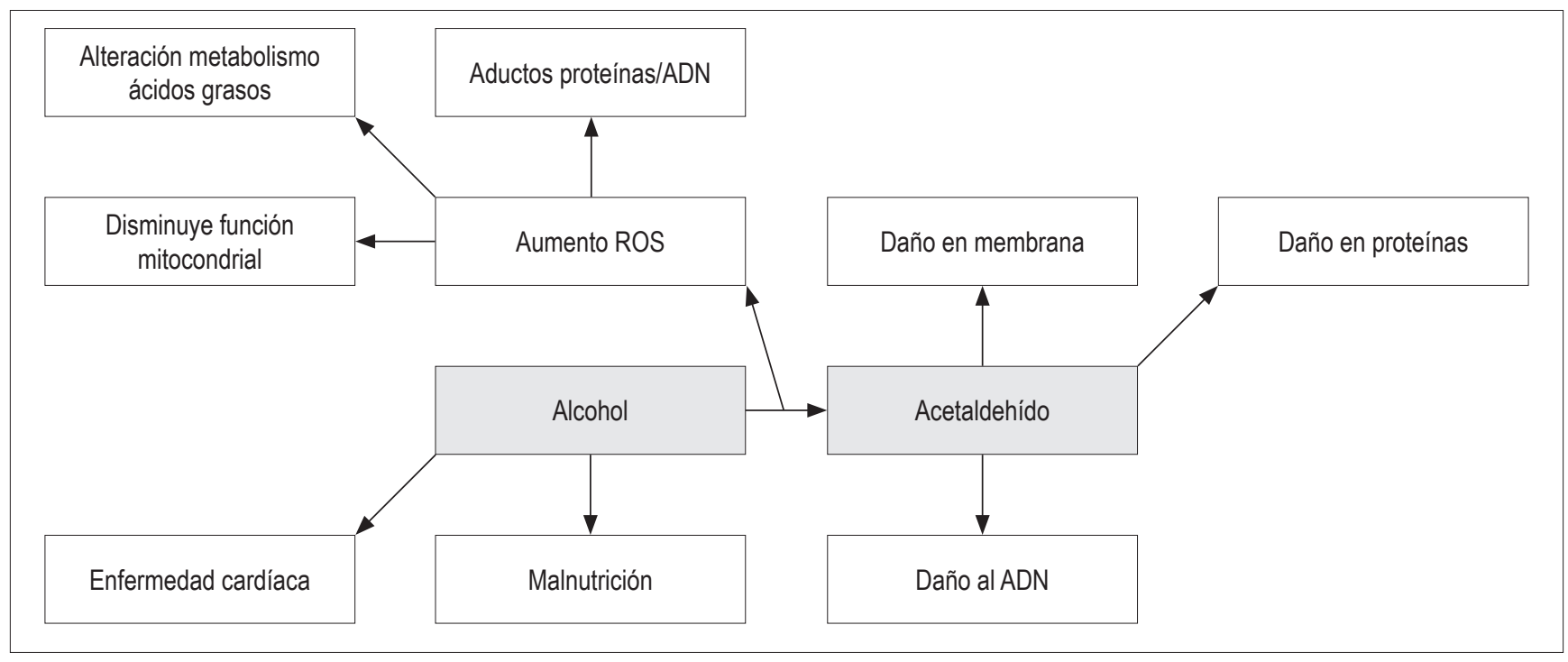

Figura 3. Efectos tóxicos del metabolismo del alcohol.

los hepatocitos; a su vez, las macrovacuolas pueden desplazar el núcleo a la periferia de la célula. La esteatosis puede empeorar con la inflamación y presencia de infiltrados de polimorfonucleares y leucocitos constituyendo el siguiente estadio, denominado esteatohepatitis $(15,55)$. En esta enfermedad se presentan alteraciones en la estructura del tejido de localización preferente en las áreas centrolobulillares, como acumulación de ácidos grasos libres, que se observa como acumulación de gotas de grasa y donde se aprecian los infiltrados celulares mencionados anteriormente; además, hay incremento de la lipogénesis y conversión de acetil CoA a ácidos grasos $(16,55,56)$.

Otra manifestación de la enfermedad hepática por alcohol es la hepatitis alcohólica, caracterizada por necrosis celular con infiltrado de leucocitos polimorfonucleares y degeneración del hepatocito localizada en el nivel del centrolobulillar (56). La muerte celular de hepatocitos antes mencionada, inducida por el consumo de etanol, puede desencadenar una fibrosis hepática y producir una regeneración fibrótica con acumulación de colágeno tipo I, debido al incremento en la síntesis de este tipo de colágeno por las células hepáticas estrelladas (HSCs) y a la mayor producción de proteínas de la matriz extracelular, como osteopontina. Estas HSCs pueden activarse por hepatocitos con daño celular y a la vez incrementar la síntesis de inhibidores de colagenasas, activar células de Kupffer y neutrófilos, lo que lleva a un daño hepático crónico por el incremento de factores de crecimiento, citocinas, quimiocinas y radicales libres que llevan a retroalimentar la activación de las HSCs y a aumentar sus efectos, llevando a mayores complicaciones en el hígado, como cirrosis $(16,57)$.

\section{POLIMORFISMOS Y SUSCEPTIBILIDAD GENÉTICA EN EL DESARROLLO DE CIRROSIS}

En el año de 1965, Von Wartburg, Papenberg y Aebi reportaron que ciertos individuos presentaban una forma atípica de la enzima alcohol deshidrogenasa (58), y Greenfield y Pietruzko reportaron 2 isoenzimas de ALDH que tenían diferente actividad metabólica (59).

Se demostró actividad metabólica disminuida de $\mathrm{ADH}$ en población caucásica. Al analizar grupos de estos pacientes con cirrosis alcohólica, cirrosis no alcohólica, bebedores crónicos sin enfermedad del hígado y bebedores moderados sin enfermedad del hígado se encontró que la actividad de la $\mathrm{ADH}$ es menor en pacientes con cirrosis alcohólica; igualmente, esta actividad se encontró disminuida en pacientes cirróticos y se asoció con la presencia del polimorfismo $\mathrm{ADH} 1 \mathrm{~B} 1$ versus la presencia del polimorfismo $\mathrm{ADH} 1 \mathrm{~B} 2$. A su vez, en los pacientes con consumo crónico de alcohol la actividad de ALDH se encontró disminuida, por tanto se evidencia que, de acuerdo con la expresión génica, se pueden observar diferentes efectos en la eficiencia del metabolismo (29).

Se ha descrito mutación del gen ALDH en población japonesa, que codifica para una forma inactiva de la enzima y ocasiona una respuesta denominada Oriental flushing response: los pacientes con esta mutación presentan cefalea, náuseas y mareo, lo que obliga a disminuir el consumo de alcohol (60). Sin embargo, son estudios poco concluyentes porque también se ha descrito aumento de la actividad de la enzima $\mathrm{ADH}\left(\mathrm{ADH} 1^{*} \mathrm{~B} 2\right)$ y disminución de la actividad de la enzima ALDH $(\operatorname{ALDH} 2 * 2)$, que genera incremento 
en la formación de acetaldehído y baja eficiencia en su oxidación, lo que ocasiona daño en el tejido celular e intoxicación; igualmente, ha sido confirmado in vitro (61). También se propone que el riesgo de desarrollar cirrosis puede estar relacionado con las interacciones entre los genes que codifican enzimas del metabolismo, es decir, la expresión del gen de ALDH con baja actividad metabólica (ALDH2*2) y las variantes $\mathrm{ADH} 1 \mathrm{~B}^{*} 2$ y $\mathrm{CYP} 2 \mathrm{E} 1{ }^{*} 5 \mathrm{~B}$ ocasionan mayor producción de acetaldehído y un metabolismo lento, que serían responsables del daño a las células (62); contrario a estos resultados, otro estudio encontró mayor incidencia de la variante $\mathrm{ALDH} 2{ }^{*} 1$ en pacientes con cirrosis alcohólica (63). En la tabla 3 se resumen algunos de los estudios de polimorfismos de los genes ADH, CYP2E1 y ALDH que se han relacionado con el desarrollo de enfermedades alcohólicas hepáticas.

\section{CONCLUSIÓN}

El etanol es un importante tóxico celular que genera diferentes tipos de sustancias, como el metabolito acetaldehído y ROS, que causan daños directos en la célula, y activación de células estrelladas del hígado con producción anormal de colágeno y cambio en la estructura del hígado, ocasionando una fibrosis que puede evolucionar en cirrosis. Existe evidencia que la combinación de los polimorfismos en los genes que codifican para las enzimas que metabolizan el alcohol, como $\mathrm{ADH}_{1} \mathrm{~B}^{*} 2, \mathrm{ADH}_{1} \mathrm{C}^{*} 1, \mathrm{ALDH} 2 * 2$ y CYP2E1*5, incrementan la susceptibilidad de desarrollar la enfermedad hepática por la acumulación de acetaldehído en el organismo.

El presente reporte indica la necesidad de continuar desarrollando estudios sobre la toxicidad del alcohol en el organismo, teniendo en cuenta el genotipo presente para las enzimas que metabolicen el etanol y que a futuro contribuyan con el pronóstico de la capacidad de un individuo para el eliminar el contenido del etanol en el organismo.

\section{Fuentes de financiación}

Esta publicación hace parte del proyecto 111556935008 financiado por el Departamento Administrativo de Ciencia, Tecnología e Innovación - COLCIENCIAS y la Vicerrectoría de Investigación, Universidad de Antioquia (Proyecto Sostenibilidad).

Tabla 3. Estudios de polimorfismos en los genes $\mathrm{ADH}$ y ALDH presentes en diferentes poblaciones.

\begin{tabular}{|c|c|c|c|}
\hline Referencia & Tipo de estudio & Población de estudio & Resultados \\
\hline Muramatsu, et al (60) & Corte transversal & $\begin{array}{l}\text { Población china, } 32 \text { alcohólicos y } 105 \text { no } \\
\text { alcohólicos }\end{array}$ & $\begin{array}{l}\text { Alcohólicos tienen baja frecuencia del genotipo } \\
\text { ADH1 }^{*} 2(0,25) \text { y ALDH2*2 }(0,34) \text { comparado con } \\
\text { la frecuencia de los controles }(0,41 \text { y } 0,48) \text {. No } \\
\text { hay diferencias estadísticamente significativas }\end{array}$ \\
\hline Poupon, et al (29) & Corte transversal & $\begin{array}{l}\text { Población caucásica. Pacientes con cirrosis } \\
\text { alcohólica }(n=31) \text {, cirrosis no alcohólica }(n=25) \text {, } \\
\text { alcohólicos sin enfermedad del hígado }(n=62) \\
\text { y bebedores moderados sin enfermedad del } \\
\text { hígado ( } n=43) \text {, población caucásica }\end{array}$ & $\begin{array}{l}\text { No hay asociación entre ADH1C con cirrosis } \\
\text { alcohólica. } \\
\text { La actividad catalítica de ALDH no fue afectada } \\
\text { entre los diferentes pacientes }\end{array}$ \\
\hline Yoshida, et al (38) & $\begin{array}{l}\text { Estudios } \\
\text { de casos } \\
\text { y controles }\end{array}$ & $\begin{array}{l}\text { Población japonesa. Pacientes con enfermedad } \\
\text { hepática por alcohol }(E H A)(n=23) \text { y controles } \\
\text { sin EHA }(n=49)\end{array}$ & $\begin{array}{l}\text { No se encontraron diferencias entre sujetos con } \\
\text { EHA y controles con la frecuencia de ADH1B2 }\end{array}$ \\
\hline Khan, et al (62) & $\begin{array}{l}\text { Estudio de casos y } \\
\text { controles }\end{array}$ & $\begin{array}{l}\text { Población India. Pacientes con cirrosis alcohólica } \\
(n=175) \text {, cirrosis no alcohólica }(n=140) \text {, } \\
\text { bebedores con alto consumo }(n=140) \text { y no } \\
\text { bebedores }(n=255)\end{array}$ & 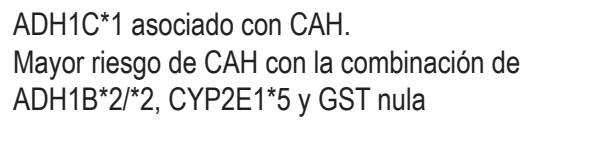 \\
\hline
\end{tabular}

ADH1: alcohol deshidrogenasa; CYP2E1: citocromo p450; GST: glutatión S transferasa; AH: cirrosis alcohólica de hígado.

\section{REFERENCIAS}

1. Organization WH. Global status report on alcohol and health. Geneva: World Health Organization 2014 [citado 9 de septiembre de 2014]. Available from: http://www.who. int/iris/bitstream/10665/112736/1/9789240692763
eng.pdf?ua=1Available from: http://www.who.int/substance_abuse/publications/global_alcohol_report/en/

2. Rehm J, Mathers C, Popova S, Thavorncharoensap M, Teerawattananon Y, Patra J. Global burden of disease and injury and economic cost attributable to alcohol use and alcohol-use disorders. Lancet. 2009;373(9682):2223-33. 
3. Zakhari S. Overview: How is alcohol metabolized by the body? Alcohol Res Health. 2006;29(4):245-54.

4. Homann N, Stickel F, Konig IR, Jacobs A, Junghanns K, Benesova $\mathrm{M}$, et al. Alcohol dehydrogenase $1 \mathrm{C}^{*} 1$ allele is a genetic marker for alcohol-associated cancer in heavy drinkers. Int J Cancer. 2006;118(8):1998-2002.

5. Yokoyama A, Mizukami T, Matsui T, Yokoyama T, Kimura M, Matsushita S, et al. Genetic Polymorphisms of Alcohol Dehydrogenase-1B and Aldehyde Dehydrogenase-2 and Liver Cirrhosis, Chronic Calcific Pancreatitis, Diabetes Mellitus, and Hypertension Among Japanese Alcoholic Men. Alcohol Clin Exp Res. 2013;37(8):1391-401.

6. Wang Y, Millonig G, Nair J, Patsenker E, Stickel F, Mueller $S$, et al. Ethanol-induced cytochrome P4502E1 causes carcinogenic etheno-DNA lesions in alcoholic liver disease. Hepatology. 2009;50(2):453-61.

7. Baraona E, Lieber CS. Effects of ethanol on lipid metabolism. J Lipid Res. 1979;20(3):289-315.

8. Guynn RW, Pieklik JR. Dependence on dose of the acute effects of ethanol on liver metabolism in vivo. J Clin Invest. 1975;56(6):1411-9.

9. Lundquist F, Tygstrup N, Winkler K, Jensen KB. Glycerol metabolism in the human liver: Inhibition by ethanol. Science. 1965;150(3696):616-7.

10. Korsten MA, Matsuzaki S, Feinman L, Lieber CS. High blood acetaldehyde levels after ethanol administration. Difference between alcoholic and nonalcoholic subjects. N Engl J Med. 1975;292(8):386-9.

11. Minana JB, Gomez-Cambronero L, Lloret A, Pallardo FV, Del Olmo J, Escudero A, et al. Mitochondrial oxidative stress and CD95 ligand: A dual mechanism for hepatocyte apoptosis in chronic alcoholism. Hepatology. 2002;35(5):1205-14.

12. Lefevre AF, DeCarli LM, Lieber CS. Effect of ethanol on cholesterol and bile acid metabolism. J Lipid Res. 1972;13(1):48-55.

13. Crouse JR, Gerson CD, DeCarli LM, Lieber CS. Role of acetate in the reduction of plasma free fatty acids produced by ethanol in man. J Lipid Res. 1968;9(4):509-12.

14. Riveros-Rosas H, Julian-Sanchez A, Pina E. Enzymology of ethanol and acetaldehyde metabolism in mammals. Arch Med Res. 1997;28(4):453-71.

15. Rehm J, Shield K. Global Alcohol-Attributable deaths From Cancer, Liver Cirrhosis, and Injury in 2010 Alcohol Res Curr Rev. 2014;35(2): 10.

16. Orman ES, Odena G, Bataller R. Alcoholic liver disease: Pathogenesis, management, and novel targets for therapy. J Gastroenterol Hepatol. 2013;28 Suppl 1:77-84.

17. Donato F, Tagger A, Gelatti U, Parrinello G, Boffetta P, Albertini A, et al. Alcohol and hepatocellular carcinoma: the effect of lifetime intake and hepatitis virus infections in men and women. Am J Epidemiol. 2002;155(4):323-31.

18. Bellentani S, Saccoccio G, Costa G, Tiribelli C, Manenti F, Sodde M, et al. Drinking habits as cofactors of risk for alcohol induced liver damage. The Dionysos Study Group. Gut. $1997 ; 41(6): 845-50$.
19. Frezza M, di Padova C, Pozzato G, Terpin M, Baraona E, Lieber CS. High blood alcohol levels in women. The role of decreased gastric alcohol. N Engl J Med. 1990;322(2):95-9.

20. Hoyumpa AM, Jr., Nichols S, Henderson GI, Schenker S. Intestinal thiamin transport: Effect of chronic ethanol administration in rats. Am J Clin Nutr. 1978;31(6):938-45.

21. Lieber CS, DeCarli LM. An experimental model of alcohol feeding and liver injury in the baboon. J Med Primatol. 1974;3(3):153-63.

22. Lieber CS, Robins SJ, Li J, DeCarli LM, Mak KM, Fasulo JM, et al. Phosphatidylcholine protects against fibrosis and cirrhosis in the baboon. Gastroenterology. 1994;106(1):152-9.

23. Lieber CS, DeCarli LM, Mak KM, Kim CI, Leo MA. Attenuation of alcohol-induced hepatic fibrosis by polyunsaturated lecithin. Hepatology. 1990;12(6):1390-8.

24. Hrubec Z, Omenn GS. Evidence of genetic predisposition to alcoholic cirrhosis and psychosis: twin concordances for alcoholism and its biological end points by zygosity among male veterans. Alcohol Clin Exp Res. 1981;5(2):207-15.

25. Reed T, Page WF, Viken RJ, Christian JC. Genetic Predisposition to Organ-Specific Endpoints of Alcoholism. Alcohol Clin Exp Res. 1996;20(9):1528-33.

26. Marrero JA, Fontana RJ, Fu S, Conjeevaram HS, Su GL, Lok AS. Alcohol, tobacco and obesity are synergistic risk factors for hepatocellular carcinoma. J Hepatol. 2005;42(2):218-24.

27. Osier MV, Pakstis AJ, Soodyall H, Comas D, Goldman D, Odunsi A, et al. A global perspective on genetic variation at the $\mathrm{ADH}$ genes reveals unusual patterns of linkage disequilibrium and diversity. Am J Hum Genet. 2002;71(1):84-99.

28. Hoog JO, Ostberg LJ. Mammalian alcohol dehydrogenases: A comparative investigation at gene and protein levels. Chem Biol Interact. 2011;191(1-3):2-7.

29. Poupon RE, Nalpas B, Coutelle C, Fleury B, Couzigou $\mathrm{P}$, Higueret D. Polymorphism of alcohol dehydrogenase, alcohol and aldehyde dehydrogenase. Hepatology. $1992 ; 15(6): 1017-22$.

30. Carrigan MA, Uryasev O, Davis RP, Zhai L, Hurley TD, Benner SA. The natural history of class I primate alcohol dehydrogenases includes gene duplication, gene loss, and gene conversion. PLoS One. 2012;7(7):e41175.

31. Stewart MJ, McBride MS, Winter LA, Duester G. Promoters for the human alcohol dehydrogenase genes $\mathrm{ADH} 1, \mathrm{ADH} 2$, and $\mathrm{ADH} 3$ : Interaction of CCAAT/enhancer-binding protein with elements flanking the $\mathrm{ADH} 2$ TATA box. Gene. 1990;90(2):271-9.

32. Vallee BL, Bazzone TJ. Isozymes of human liver alcohol dehydrogenase. Isozymes Curr Top Biol Med Res. 1983;8:219-44.

33. Duester G, Farres J, Felder MR, Holmes RS, Hoog JO, Pares $\mathrm{X}$, et al. Recommended nomenclature for the vertebrate alcohol dehydrogenase gene family. Biochem Pharmacol. 1999;58(3):389-95.

34. Persson B, Hedlund J, Jornvall H. Medium- and short-chain dehydrogenase/reductase gene and protein families. Cell Mol Life Sci. 2008;65(24):3879-94. 
35. Smith M, Hopkinson DA, Harris H. Alcohol dehydrogenase isozymes in adult human stomach and liver: evidence for activity of the $\mathrm{ADH} 3$ locus. Ann Hum Genet. 1972;35(3):243-53.

36. Smith M, Hopkinson DA, Harris H. Developmental changes and polymorphism in human alcohol dehydrogenase. Ann Hum Genet. 1971;34(3):251-71.

37. Birley AJ, James MR, Dickson PA, Montgomery GW, Heath AC, Martin NG, et al. ADH single nucleotide polymorphism associations with alcohol metabolism in vivo. Hum Mol Genet. 2009;18(8):1533-42.

38. Yoshida A, Shibuya A. Polymorphisms of Alcohol and Aldehyde Dehydrogenases and Their Significance for Alcohol Liver Diseases. In: Watson R, editor. Liver Pathology and Alcohol. Drug and Alcohol Abuse Reviews. 2: Humana Press; 1991. p. 441-66.

39. Black WJ, Stagos D, Marchitti SA, Nebert DW, Tipton KF, Bairoch A, et al. Human aldehyde dehydrogenase genes: Alternatively spliced transcriptional. Pharmacogenet Genomics. 2009;19(11):893-902.

40. Hsu LC, Yoshida A, Mohandas T. Chromosomal assignment of the genes for human aldehyde dehydrogenase- 1 and aldehyde dehydrogenase-2. Am J Hum Genet. 1986;38(5):641-8.

41. Hsu LC, Bendel RE, Yoshida A. Genomic structure of the human mitochondrial aldehyde dehydrogenase gene. Genomics. 1988;2(1):57-65.

42. Crabb DW, Matsumoto M, Chang D, You M. Overview of the role of alcohol dehydrogenase and aldehyde dehydrogenase and their variants in the genesis of alcohol-related pathology. Proc Nutr Soc. 2004;63(1):49-63.

43. Farres J, Wang X, Takahashi K, Cunningham SJ, Wang T, Weiner $\mathrm{H}$. Effects of changing glutamate 487 to lysine in rat and human liver mitochondrial aldehyde dehydrogenase. A model to study human (Oriental type) class 2 aldehyde dehydrogenase. J Biol Chem. 1994;269(19):13854-60.

44. Umeno M, McBride OW, Yang CS, Gelboin HV, Gonzalez FJ. Human ethanol-inducible P450IIE1: Complete gene sequence, promoter characterization, chromosome mapping, and cDNA-directed expression. Biochemistry. 1988;27(25):9006-13.

45. Ravindranath V, Anandatheerthavarada HK, Shankar SK. NADPH cytochrome P-450 reductase in rat, mouse and human brain. Biochem Pharmacol. 1990;39(6):1013-8.

46. Shen Z, Wells RL, Liu J, Elkind MM. Identification of a cytochrome P450 gene by reverse transcription--PCR using. Proc Natl Acad Sci USA. 1993;90(24):11483-7.

47. Lieber CS. Ethanol metabolism, cirrhosis and alcoholism. Clin Chim Acta. 1997;257(1):59-84.

48. $\mathrm{Hu} \mathrm{Y}$, Ingelman-Sundberg $\mathrm{M}$, Lindros $\mathrm{KO}$. Induction mechanisms of cytochrome P450 2E1 in liver: Interplay between ethanol treatment and starvation. Biochem Pharmacol. 1995;50(2):155-61.

49. Albano E, Vidali M. Immune mechanisms in alcoholic liver disease. Genes Nutr. 2010;5(2):141-7.

50. Bell GI, Najarian RC, Mullenbach GT, Hallewell RA. cDNA sequence coding for human kidney catalase. Nucleic Acids Res. 1986;14(13):5561-2.

51. Koechling UM, Amit Z. Relationship between blood catalase activity and drinking history in a human. Alcohol. 1992;27(2):181-8.

52. Wen JK, Osumi T, Hashimoto T, Ogata M. Molecular analysis of human acatalasemia. Identification of a splicing mutation. J Mol Biol. 1990;211(2):383-93.

53. Kishimoto Y, Murakami Y, Hayashi K, Takahara S, Sugimura $\mathrm{T}$, Sekiya T. Detection of a common mutation of the catalase gene in Japanese acatalasemic patients. Hum Genet. 1992;88(5):487-90.

54. Góth L, Shemirani A, Kalmár T. A Novel Catalase Mutation (a GA Insertion) Causes the Hungarian Type of Acatalasemia. Blood Cell Mol Dis. 2000;26(2):151-4.

55. Farfan Labonne BE, Gutierrez M, Gomez-Quiroz LE, Konigsberg Fainstein M, Bucio L, Souza V, et al. Acetaldehyde-induced mitochondrial dysfunction sensitizes hepatocytes to oxidative damage. Cell Biol Toxicol. 2009;25(6):599-609.

56. Pares A, Caballería J. Patología orgánica. Adicciones. 2002;14:17.

57. Gao B, Bataller R. Alcoholic liver disease: Pathogenesis and new therapeutic targets. Gastroenterology. 2011; 141(5): 1572-85.

58. Von Wartbung JP, Papenberg J, Aebi H. An atypical human alcohol dehydrogenase. Can J Biochem. 1965:43,889.

59. Greenfield NJ, Pietruszko R. Two aldehyde dehydrogenases from human liver. Isolation via affinity chromatography and characterization of the isozymes. Biochim Biophys Acta. 1977:483:35-45.

60. Harada S, Misawa S, Agarwal DP, Goedde HW. Liver alcohol dehydrogenase and aldehyde dehydrogenase in the Japanese: Isozyme. Am J Hum Genet. 1980;32(1):8-15.

61. Khan AJ, Husain Q, Choudhuri G, Parmar D. Association of polymorphism in alcohol dehydrogenase and interaction with other. Drug Alcohol Depen. 2010;109(1-3):190-7.

62. Tanaka F, Shiratori Y, Yokosuka O, Imazeki F, Tsukada Y, Omata $\mathrm{M}$. High incidence of $\mathrm{ADH} 2{ }^{*} 1 / \mathrm{ALDH} 2 * 1$ genes among Japanese alcohol dependents and patients with alcoholic liver disease. Hepatology. 1996;23:234-9.

63. Muramatsu T, Wang ZC, Fang YR, Hu KB, Yan H, Yamada $\mathrm{K}$, et al. Alcohol and aldehyde dehydrogenase genotypes and drinking behavior of Chinese living in Shanghai. Hum Genet. 1995;96(2):151-4. 